\title{
INVARIANT DIFFERENTIAL EQUATIONS ON HOMOGENEOUS MANIFOLDS
}

\author{
BY SIGURDUR HELGASON ${ }^{1}$
}

1. Historical origins of Lie group theory. Nowadays when Lie groups enter in a profound way into so many areas of mathematics, their historical origin is of considerable general interest. The connection between Lie groups and differential equations is not very pronounced in the modern theory of Lie groups, so in this introduction we attempt to describe some of the foundational work of S. Lie, W. Killing and É. Cartan at the time when the interplay with differential equations was significant. In fact, the actual construction of the exceptional simple Lie groups seems to have been accomplished first by means of differential equations.

Although motion groups in $\mathbf{R}^{3}$ had occurred in the work of $\mathbf{C}$. Jordan prior to 1870 , Lie group theory as a general structure theory for the transformation groups themselves originated around 1873 with Lie's efforts about that time to use group theoretic methods on differential equations as suggested by Galois' theory for algebraic equations. It seems that a lecture by Sylow in 1863 (when Lie was 20) on Galois theory ${ }^{2}$ (Lie and Engel [9, vol. 3, p. XXII]) and his collaboration with F. Klein, 1870, on curves and transformations (Klein and Lie [6], Engel [3b, p. 35]) were particularly instrumental in suggesting to him the following:

Problem (LIE [8a]). Given a system of differential equations how can knowledge about its invariance group be utilized towards its integration?

Since the solutions of a differential equation are functions, not just numbers as for an algebraic equation, one can take two different viewpoints for an analogy with Galois theory.

Analytic viewpoint (Lie (1871-1874)). For a system of differential equations, consider the group of diffeomorphisms of the underlying space leaving the system stable (i.e., permuting the solutions).

Algebraic viewpoint (Picard (1883), Vessiot (1891)). For a given differential equation consider the group of automorphisms of the field generated by the solutions, fixing the elements of the coefficient field.

To indicate the flavor of the resulting theories I just recall a couple of the best known results. In ordinary Galois theory one has the fundamental result that an algebraic equation is solvable by radicals if and only if the Galois group is solvable. In the Picard-Vessiot theory one introduces similarly the

An expanded version of an address given at the annual meeting of the American Mathematical Society in Washington, D. C. on January 23, 1975; received by the editors October 20, 1976.

AMS (MOS) subject classifications (1970). Primary 22E30, 35C15, 43A85, 58G99; Secondary 22-03, 22-02, 43A90.

1 Partially supported by NSF Grant MCS 75-21415.

${ }^{2}$ See "Sources" at the end of this section. 
Galois group of a linear homogeneous ordinary differential equation, the so-called differential Galois group (since the automorphisms are assumed to commute with differentiation). The solvability of this group is then necessary and sufficient for the equation to be solvable by quadratures, $f(x) \rightarrow$ $\int f(x) d x$, and exponentiation, $g(x) \rightarrow e^{g(x)}$.

To indicate the rudiments of Lie's theory, consider a differential equation

$$
d y / d x=Y(x, y) / X(x, y)
$$

in the plane. It is called stable under a 1-parameter group $\phi_{t}(t \in \mathbf{R})$ of diffeomorphisms if each $\phi_{t}$ permutes the integral curves (all concepts are here local).

EXAMPLE.

$$
\frac{d y}{d x}=\frac{y+x\left(x^{2}+y^{2}\right)}{x-y\left(x^{2}+y^{2}\right)} .
$$

The equation can be written

$$
\left(\frac{d y}{d x}-\frac{y}{x}\right) /\left(1+\frac{y}{x} \frac{d y}{d x}\right)=x^{2}+y^{2}
$$

and since the left-hand side is the tangent of the angle between the integral curve and the radius vector, it is clear that the integral curves intersect each circle $x^{2}+y^{2}=r^{2}$ under a fixed angle. The group of rotations around the origin therefore permutes the integral curves, i.e., leaves the equation stable.

For a 1-parameter group $\phi_{t}$ of transformations in the plane with $\phi_{0}$ the identity let $T$ denote the induced vector field,

$$
T_{p}=\left\{\frac{d\left(\phi_{t} \cdot p\right)}{d t}\right\}_{t=0}=\xi(p) \frac{\partial}{\partial x}+\eta(p) \frac{\partial}{\partial y}, \quad p \in \mathbf{R}^{2} .
$$

THEOREM 1.1 (LIE [8a]). Equation (1) is stable under $\phi_{t}$ if and only if the vector field $Z=X \partial / \partial x+Y \partial / \partial y$ satisfies

$$
[T, Z]=\lambda Z
$$

where $\lambda$ is a function. In this case $(X \eta-Y \xi)^{-1}$ is an integrating factor for the equation $X d y-Y d x=0$.

Thus, knowing a stability group for a differential equation provides a way to solve it. For the example above, equation (2) is stable under the group

$$
\phi_{t}:(x, y) \rightarrow(x \cos t-y \sin t, x \sin t+y \cos \imath)
$$

for which

$$
T=-y \frac{\partial}{\partial x}+x \frac{\partial}{\partial y}
$$

and the theorem gives the solution $y=x \tan \left(\frac{1}{2}\left(x^{2}+y^{2}\right)+C\right), C$ a constant.

Generalizing the 1-parameter group $\left(\phi_{t}\right)$ above, let

$$
x_{i}^{\prime}=f_{i}\left(x_{1}, \ldots, x_{n} ; t_{1}, \ldots, t_{r}\right)
$$

be an $r$-parameter local transformation group of $n$-space, the origin $\left(t_{1}, \ldots, t_{r}\right)=(0, \ldots, 0)$ representing the identity transformation and rank $\left(\partial f_{i} / \partial t_{k}\right)=r$. In analogy with (3) define the vector fields ("infinitesimal 
transformations")

$$
T_{k}=\sum_{i=1}^{n}\left(\frac{\partial f_{i}}{\partial t_{k}}\right)_{t=0} \frac{\partial}{\partial x_{i}} \quad(1 \leqslant k \leqslant r)
$$

on $\mathbf{R}^{n}$. Passing to the second derivatives of the $f_{i}$, Lie proved that the group law for (5) implies that

$$
\left[T_{k}, T_{l}\right]=\sum_{p=1}^{r} C_{k l}^{p} T_{p}
$$

where the $C_{k l}^{p}$ are constants satisfying

$$
C_{k l}^{p}=-C_{l k}^{p}, \quad \sum_{q=1}^{r}\left(C_{k q}^{p} C_{l m}^{q}+C_{m q}^{p} C_{k l}^{q}+C_{l q}^{p} C_{m k}^{q}\right)=0 .
$$

This device, which through Lie's so-called "three fundamental theorems" relates the study of the local transformation group (5) to the algebraic study of the Lie algebra (6), (7) (the third theorem amounts to the statement that every system of constants $C_{k l}^{p}$ satisfying (7) arises in this way), forms the foundation of Lie's theory of transformation groups. His first proof of (6) (cf. [8c, p. 52]) was incomplete (cf. loc. cit., p. 617) but was completed in his paper [8d, p. 462]; in the modern theory of Lie groups relation (6) amounts to the fact that when a Lie group $G$ acts on a manifold $M$ there is induced a homomorphism of the corresponding Lie algebra $g$ into the Lie algebra of vector fields on $M$.

In one generalization of Theorem 1.1 from $\mathbf{R}^{2}$ to $\mathbf{R}^{n}$ (cf. Lie [8b]) one considers a differential equation

$$
\sum_{i=1}^{n} X_{i} \frac{\partial f}{\partial x_{i}}=0
$$

where $X_{i} \in C^{\infty}\left(\mathbf{R}^{n}\right)$. Assuming the equation stable under a solvable $(n-1)$ parameter transformation group, its solutions can be found by quadratures. (Here the term "solvable" has replaced the older term "integrable" and the term quadrature is used for integration $f(x) \rightarrow \int f(x) d x$ in analogy with taking square roots in the analogous result for an algebraic equation.)

Such results, and their generalization to systems, suggested the problem of classifying all local transformation groups of $\mathbf{R}^{n}$. Lie solved this for $n=1$, where the local groups are

$$
\begin{gathered}
x \rightarrow x+a, \quad a \in \mathbf{R} \text { const, } \\
x \rightarrow a x+b, \quad a \in \mathbf{R}-(0), b \in \mathbf{R}, \\
x \rightarrow(a x+b) /(c x+d), \quad a, b, c, d \in \mathbf{R}, a d-b c=1 .
\end{gathered}
$$

For $n=2$ the possibilities are already quite numerous (cf. [8f, vol. V, p. 768]) so the assumption of primitivity (no invariant decomposition of the space into lower-dimensional submanifolds) was introduced. Then there are for $n=2$ just 3 possibilities, namely the 8-parameter analog of (11) (the projective group), the 6-parameter analog of (10) (the affine group), and its 5-parameter subgroup of area preserving transformations (analog of (9), the special affine group). ${ }^{3}$ Lie [8e] and, his student in Leipzig, Page [11] settled the cases $n=3$,

\footnotetext{
${ }^{3}$ In [10] Mostow determines all global transitive Lie transformation groups of surfaces.
} 
4 respectively; later Kowalewski and Beutner worked out the cases $n=5$ and $n=6$, respectively. The direct attack on the problem was not continued further because the complexities had become rather formidable, and since the problem had taken a new direction through the work of Killing and Cartan.

W. Killing, who wrote his dissertation with Weierstrass in 1872, began in 1877-1878 geometric investigations which, without knowledge of Lie's work, led him to concepts close to Lie's infinitesimal transformations and to relations equivalent to (6). (Cf. Lie and Engel [9, vol. 3, p. 768].) Even before getting acquainted with Lie's work, Killing had set himself the problem of finding all possible "Zusammensetzungen" of $r$-parameter groups. In other words, he wanted to find all possible ways, up to isomorphism, in which an $r$-dimensional vector space can be turned into a Lie algebra.

Thus Lie's classification problem (which, as indicated, arose from his study of differential equations) consisted of Killing's algebraic problem together with the problem of classifying the various representations of a given group as a transformation group. This viewpoint was decisive for the theory of Lie groups, but separated it gradually from differential equations.

On 18 October 1887 Killing wrote to F. Engel, who was then Lie's assistant in Leipzig, that he had succeeded in finding a complete classification of the simple Lie algebra $\mathfrak{g}$ over $\mathbf{C}$. In this work [5] Killing introduced many of the fundamental concepts for the theory of simple Lie algebras, in particular, the following:

(a) The rank $l$ of $g$.

(b) For the linear transformation ad $X: Y \rightarrow[X, Y]$ the characteristic equation

(12) $\operatorname{det}(\omega-\operatorname{ad} X) \equiv \omega^{r}-\psi_{2}(X) \omega^{r-2}+\cdots \pm \psi_{r-l}(X) \omega^{l}=0$.

The coefficient $2 \psi_{2}(X)$ which equals $\operatorname{Trace}(\operatorname{ad} X)^{2}$ is now called the Killing form. Equation (12) had also been used extensively by Lie.

(c) The roots of $\mathfrak{g}$ which, by definition, are the functions $\omega(X)$ on $\mathfrak{g}$ satisfying (12).

(d) A basis $\omega_{1}, \ldots, \omega_{l}$ of roots of which all roots are integral linear combinations (with all coefficients of the same sign) and the associated matrix $\left(a_{i j}\right)$ where

$$
-a_{i j}=\text { the largest integer } q \text { such that } \omega_{j}+q \omega_{i} \text { is a root. }
$$

This matrix is now called the Cartan matrix.

In this remarkable work, Killing finds all possibilities for the matrix $\left(a_{i j}\right)$ and writes down the corresponding roots $\omega(X)$ (cf. [5, II, §15]). Thus he arrives at the statement that apart from the classical simple Lie algebras

$$
A_{l}(l \geqslant 1), \quad B_{l}(l \geqslant 2), \quad C_{l}(l \geqslant 3), \quad D_{l}(l \geqslant 3)
$$

(known from Lie's work), there are only six more, of ranks and dimension, respectively,

$$
\begin{aligned}
& l=2,4,4,6,7,8, \\
& r=14,52,52,78,133,248 .
\end{aligned}
$$

These exceptional Lie algebras are denoted $G_{2}, E_{4}, F_{4}, E_{6}, E_{7}, E_{8}$, respectively. Killing denoted $G_{2}$ by (IIC); he observed that $A_{3}=D_{3}$, but did not notice that $E_{4}=F_{4}$, although, as Cartan remarked, this is immediate from his 
root tables in [5, II, pp. 30-31]. Killing's work [5] was immediately recognized as a breakthrough. However, it was also criticized, particularly by Lie $[\mathbf{9}$, vol. 3, pp. 768-771], for serious gaps and inaccuracies. É. Cartan set himself the problem of giving genuine proofs of the results stated by Killing. Apart from pointing out many errors in detail, Cartan found the following gaps in Killing's papers particularly significant: (a) In [5, II] Killing makes the explicit assumption that the nonzero roots of (12) are simple. While this is correct, his justification in [5, III] of this assumption was incorrect. (b) A proof that at most one $\mathfrak{g}$ can correspond to a given matrix $\left(a_{i j}\right)$ was lacking. Cartan showed, case-by-case, that this is so; an a priori proof was given much later by van der Waerden [13].

The actual existence of the exceptional Lie algebras is another major weakness of Killing's work. He indicates in [5, II, \$18] how the structural constants $C_{j k}^{i}$ can be determined from his root tables. Then the Jacobi identity (7) has to be verified; Killing does this for $G_{2}$, but for the others his indications [5, II, p. 48] seem unconvincing.

In his thesis [1b], É. Cartan gave a complete proof of the classification results stated by Killing; in outline his method follows Killing's program. He determined the matrices $\left(a_{i j}\right)$, the roots $\omega(X)$ and a basis for each of the exceptional Lie algebras with respect to which the structural constants have a simple and symmetric form [1b, $\$ \S 18-20]$ whereby the Jacobi identity (7) is (presumably) simple to verify. ${ }^{4}$ But he was also interested in realizing the exceptional Lie groups by transformations, like e.g. the classical algebra $C_{l}$ is the Lie algebra of the linear group leaving invariant the Pfaffian form

$$
x_{1} d y_{1}-y_{1} d x_{1}+\cdots+x_{l} d y_{l}-y_{l} d x_{l} \text {. }
$$

Killing had been led to expect that $G_{2}$ could be realized as a transformation group in $\mathbf{R}^{5}$, but not in a lower-dimensional space. Engel and Cartan showed that it can be realized as the stability group of the system

$$
\begin{aligned}
& d x_{3}+x_{1} d x_{2}-x_{2} d x_{1}=0, \\
& d x_{4}+x_{3} d x_{1}-x_{1} d x_{3}=0, \\
& d x_{5}+x_{2} d x_{3}-x_{3} d x_{2}=0,
\end{aligned}
$$

in $\mathbf{R}^{5}$ (Engel [3a], Cartan [1b, p. 281], Lie and Engel [9, vol. 3, p. 764]).

Cartan represented $F_{4}$ similarly by the Pfaffian system in $\mathbf{R}^{15}$ given by

$$
d z=\sum_{1}^{4} y_{i} d x_{i}, \quad d x_{i j}=x_{i} d x_{j}-x_{j} d x_{i}+y_{h} d y_{k}-y_{k} d y_{h},
$$

where $z, x_{i}, y_{j}, x_{i j}=-x_{j i}(i \neq j, i, j=1,2,3,4)$ are coordinates in $\mathbf{R}^{15}$ and in (14) $i, j, h, k$ is an even permutation [1a, p. 418]. Similar results for $E_{6}$ in $\mathbf{R}^{16}$, $E_{7}$ in $\mathbf{R}^{27}$ and $E_{8}$ in $\mathbf{R}^{29}$ as contact transformations are indicated in [1a]. Unfortunately, detailed proofs of these remarkable representations of the exceptional groups do not seem to be available.

\footnotetext{
${ }^{4}$ In [15], Witt gives an explicit geometric construction of the 5 exceptional Lie algebras and proves a priori that to each Cartan matrix $\left(a_{i j}\right)$ corresponds a simple Lie algebra (provided this is so for $l \leqslant 4$ ). Chevalley [2] indicates a general algebraic proof (without this proviso); see also Harish-Chandra [4].
} 


\section{SOURCES}

1a. É. Cartan, Über die einfachen Transformationsgruppen, Leipzig Ber., 1893, pp. 395-420; reprint, Oeuvres complètes, Vol. I, no. 1, Gauthier-Villars, Paris, 1952, pp. 107-132.

1b. __ Sur la structure des groupes de transformations finis et continus, Thèse, Paris Nony, 1894; reprint, Oeuvres complètes, Vol. I, no. 1, Groupes de Lie, Gauthier-Villars, Paris, 1952, pp. 137-287. MR 14, 343.

1c. __ Oeuvres complètes, Gauthier-Villars, Paris, 1952. MR 14, 343.

2. C. Chevalley, Sur la classification des algèbres de Lie simples et de leurs représentations, C. R. Acad. Sci. Paris 227 (1948), 1136-1138. MR 10, 280.

3a. F. Engel, Sur un groupe simple à quatorze paramètres, C. R. Acad. Sci. Paris 116 (1893), 786-788.

3b. __ Sophus Lie (obituary), Jber. Deutsch. Math.-Verein 8 (1900), 30-46.

3c. __ Wilhelm Killing (obituary), Jber. Deutsch. Math.-Verein 39 (1930), 140-154.

4. Harish-Chandra, On some applications of the universal enveloping algebra of a semisimple Lie algebra, Trans. Amer. Math. Soc. 70 (1951), 28-96. MR 13, 428.

5. W. Killing, Die Zusammensetzung der stetigen endlichen Transformationsgruppen. I, II, III, Math. Ann. 31 (1888), 252-290; ibid. 33 (1888), 1-48; ibid. 34 (1889), 57-122.

6. F. Klein and S. Lie, Uber diejenigen ebenen Kurven, welche durch ein geschlossenen System von einfach unendlich vielen vertauschbaren linearen Transformationen in sich übergehen, Math. Ann. 4 (1871), 50-84.

7. G. Kowalewski, Bestand und Wandel, Oldenbourg, München, 1950.

8a. S. Lie, Zur Theorie des Integrabilitetsfaktors, Christiania Forh. 1874, 242-254; reprint, Gessammelte Abhandlungen, Vol. III, no. XIII, pp. 176-187.

8b. , Verallgemeinerung und neue Verwertung der Jacobischen Multiplikatortheorie, Christiania Forh. 1874, 255-274; reprint, Gesammelte Abhandlungen, Vol. III, no. XIV, pp. 188-206.

8c. _ Theorie der Transformationsgruppen. II, Arch. für Math. I, 152-193, Christiania 1876; reprint, Gesammelte Abhandlungen, Vol. V., no. III, pp. 42-75.

8d. _ Theorie der Transformationsgruppen. I, Math. Ann. 16 (1880), 441-528; reprint, Gesammelte Abhandlungen, Vol. V, no. I.

8e. __ Untersuchungen über Transformationsgruppen. I, Gesammelte Abhandlungen, Vol. 5, No. XIX, pp. 453-498.

8f. __ Gesammelte Abhandlungen, 7 vols., Teubner, Leipzig, 1922-1960.

9. S. Lie and F. Engel, Theorie der Transformationsgruppen, 3 vols., Teubner, Leipzig, 1888-1893.

10. G. D. Mostow, The extensibility of local Lie groups of transformations and groups on surfaces, Ann. of Math. (2) 52 (1950), 606-636. MR 14, 18.

11. J. M. Page, On the primitive groups of transformations in space of four dimensions, Amer. J. Math. 10 (1888), 293-346.

12. E. Picard, Sur les groupes de transformation des équations différentiels linéaires, C. R. Acad. Sci. Paris 96 (1883), 1131-1134.

13. B. L. van der Waerden, Die Klassifizierung der einfachen Lie'schen gruppen, Math. Z. 37 (1933), 446-462.

14. E. Vessiot, Sur les équations différentielles linéaires, C. R. Acad. Sci. Paris 112 (1891), 778-780.

15. E. Witt, Spiegelungsgruppen und Aufzählung halbeinfacher Liescher Ringe, Abh. Math. Sem. Univ. Hamburg, 14 (1941), 289-322. MR 3, 100.

2. Invariant differential operators. In our days when Lie group theory has been so highly developed, it is reasonable to reverse the viewpoint in Lie's problem in $\$ 1$, that is, consider the group as the given object and investigate differential operators invariant under a given group. 
Let $X$ be a manifold and $D$ a differential operator on $X$. Let $\phi$ be a diffeomorphism of $X$ onto itself. For a smooth function $f$ on $X$ we put $f^{\phi}=f \circ \phi^{-1}$ and define the operator $D^{\phi}$ by

$$
D^{\phi}: f \rightarrow\left(D f^{\phi^{-1}}\right)^{\phi}
$$

then $D^{\phi}$ is another differential operator. The operator $D$ is said to be invariant under $\phi$ if

$$
D^{\phi}=D \text {. }
$$

This is a very natural condition on a differential operator and examples abound; the polynomials $P(L)$ in the Laplacian $L$ on $\mathbf{R}^{n}$ (or on any two-point homogeneous space) are precisely the differential operators invariant under all isometries; similarly the wave operator on $\mathbf{R}^{4}$ (or on an isotropic Lorentz space) is characterized by its invariance under the Poincare group (respectively, its isometry group), cf. [10a].

We shall now discuss differential operators on a manifold invariant under a transitive Lie group of diffeomorphisms. To be specific, let $G$ be a Lie group, $K \subset G$ a closed subgroup, $G / K$ the manifold of left cosets $g K(g \in G)$ and $\mathbf{D}(G / K)$ the algebra of differential operators on $G / K$ invariant under all the transformations $\tau(g): x K \rightarrow g x K$ of $G / K$ onto itself. We write $\mathbf{D}(G)$ for $\mathbf{D}(G / e)$, the algebra of left invariant differential operators on $G$. Let $g$ and $\mathfrak{f}$; respectively, denote the Lie algebras of $G$ and $K$, let $U(\mathfrak{g})$ denote the universal enveloping algebra of $\mathfrak{g}$ and $U(\mathfrak{g})^{\mathfrak{f}}$ the centralizer of $\mathfrak{t}$ in $U(\mathfrak{g})$. As noted by Schwartz and proved in $[\mathbf{8 a}$, p. 111] we have the canonical isomorphism

$$
\mathbf{D}(G) \approx U(\mathfrak{g}) .
$$

More generally [10a, Chapter III], if the coset space $G / K$ is reductive and $K$ connected, we have the isomorphism

$$
\mathbf{D}(G / K) \approx U(\mathfrak{g})^{\mathfrak{f}} /\left(U(\mathfrak{g})^{\mathfrak{f}} \cap U(\mathfrak{g})^{\mathfrak{f}}\right)
$$

expressing the algebra of invariant differential operators in Lie algebra terms.

Of the many problems one can contemplate for these operators we will discuss the following.

A. Solvability. Given $D \in \mathbf{D}(G / K)$, is the differential equation $D u=f$, for $f \in C^{\infty}(G / K)$ arbitrary, globally solvable (respectively, locally solvable)? If so we say that $D$ is globally solvable (respectively, locally solvable).

For the simplest case $G=\mathbf{R}^{n}, K=(0)$, the operators in $\mathbf{D}(G / K)$ are those of constant coefficients and the global solvability is well known (Ehrenpreis and Malgrange).

B. Joint eigenfunctions. Determine the functions on $G / K$ which are eigenfunctions of each $D \in \mathbf{D}(G / K)$. Similar problems for joint eigendistributions.

C. Eigenspace representations. Let $\mu: \mathbf{D}(G / K) \rightarrow \mathbf{C}$ be a homomorphism and let $E_{\mu}$ denote the corresponding joint eigenspace, i.e.,

$$
E_{\mu}=\left\{f \in C^{\infty}(G / K) \mid D f=\mu(D) f \text { for all } D \in \mathbf{D}(G / K)\right\}
$$


and let $T_{\mu}$ denote the natural representation of $G$ on this eigenspace, i.e.,

$$
\left(T_{\mu}(g) f\right)(x K)=f\left(g^{-1} x K\right) \text { for } g, x \in G,
$$

$f \in E_{\mu}$. For which $\mu$ is this "eigenspace representation" $T_{\mu}$ irreducible and what representations of $G$ are so obtained?

D. Extensions to vector bundles. In the following sections we survey various results concerning $\mathrm{A}, \mathrm{B}$, and $\mathrm{C}$ for certain important classes of homogeneous spaces (symmetric spaces and their duals). First we explain how invariance condition (1) can be generalized in a natural way to a smooth vector bundle $E$ over a manifold $X$ (cf. Bott [1]). If $p: E \rightarrow X$ is the projection map, a smooth section is a $C^{\infty}$ map $s: X \rightarrow E$ such that $p(s(x))=x$ for all $x \in X$. The smooth sections form a vector space $\Gamma(E)$. Let $D$ be a differential operator on $E$, that is, a linear operator from $\Gamma(E)$ to $\Gamma(E)$ which via arbitrary local trivializations of $E$ is expressed by means of ordinary partial differential operators with linear transformations of the fibers $E_{x}$ as coefficients (cf. [29, p. 66]). Let $\phi: E \rightarrow E$ be a diffeomorphism commuting with $p$ such that for each $x \in X$ the restriction map $E_{x} \rightarrow E_{\phi(x)}$ is a vector space isomorphism. Then $\phi$ acts on $\Gamma(E)$; if $s \in \Gamma(E)$ the map $s^{\phi}$ given by

$$
s^{\phi}(x)=\phi\left(s\left(\phi^{-1} x\right)\right) \quad(x \in X)
$$

obviously belongs to $\Gamma(E)$, and we can define the differential operator

$$
D^{\phi}: s \rightarrow\left(D s^{\phi^{-1}}\right)^{\phi}, \quad s \in \Gamma(E) .
$$

Again we call $D$ invariant under $\phi$ if $D^{\phi}=D$. For the trivial bundle $E=$ $X \times \mathbf{R}$ sections become functions, and this invariance notion reduces to (1).

As an example let $X=\mathbf{R}^{4}$, and $\tilde{G}$ the universal covering group of the Lorentz group $G$, and $\pi$ the natural mapping of $\tilde{G}$ onto $G ; G$ acts on $\mathbf{R}^{4}$ as the Lorentz group and $\tilde{G}$ acts on $\mathbf{R}^{4}$ via the spinor respresentation. Each $\tilde{g} \in \tilde{G}$ acts on the trivial bundle $E=\mathbf{R}^{4} \times \mathbf{R}^{4}$ by

$$
\tilde{g} \cdot(x, y)=(\pi(\tilde{g}) \cdot x, \tilde{g} \cdot y)
$$

and the Dirac operator is invariant under this action.

Now suppose $X=G / K$ and $\delta$ a representation of $K$ on a finite-dimensional vector space $V$. Let $E=G \times{ }_{K} V$ denote the product $G \times V$ modulo the equivalence relation $(g k, v) \sim(g, \delta(k) v)$ for $g \in G, k \in K, v \in V$. If $[g, v]$ denotes the equivalence class of the element $(g, v) \in G \times V$, then the mapping $p:[g, v] \rightarrow g K$ turns $E$ into a vector bundle over $X=G / K$. The group $G$ acts on $E$ via the map $g_{0} \cdot[g, v]=\left[g_{0} g, v\right]$ and this action commutes with $p$. If for a section $s \in \Gamma(E)$ we put

$$
\tilde{s}(g)=g^{-1} \cdot s(g K)
$$

then the mapping $s \rightarrow \tilde{s}$ is a bijection of $\Gamma(E)$ onto the set of smooth $V$-valued functions $f$ on $G$ satisfying $f(g k) \equiv \delta\left(k^{-1}\right)(f(g))$. Let $\mathbf{D}^{V}(G)$ denote the set of left invariant differential operators on $G$ with coefficients in $\operatorname{Hom}(V, V)$ and let $\mathbf{D}^{V}(G)^{\mathfrak{f}}$ denote the centralizer of the set $\{T+\delta(T) \mid T \in$ *) in $\mathbf{D}^{V}(G)$. Assuming $K$ compact and connected we have, in analogy with (3), that the mapping which sends $D \in D^{V}(G)^{\mathfrak{f}}$ to the differential operator $\mu(D)$ given by 


$$
(\mu(D) s)^{\tilde{}}=D \tilde{s}, \quad s \in \Gamma(E)
$$

is a homomorphism of $\mathbf{D}^{V}(G)^{\mathfrak{f}}$ onto the algebra $\mathbf{D}^{\delta}(G / K)$ of $G$-invariant differential operators on $E$.

Problems A, B, and C are meaningful for the invariant differential operators on $E$; problem $C$ is particularly interesting for the algebra $\mathbf{D}^{\delta}(G / K)$ and for subalgebras of it. As will be explained later it seems that the known irreducible representations of semisimple Lie groups and of nilpotent Lie groups can thus be realized as eigenspace representations.

For a manifold $X$ we shall use Schwartz's notation on $(X)$ and $8(X)$ for the spaces $C_{c}^{\infty}(X)$ and $C^{\infty}(X)$, respectively, with their customary topologies. Their strong duals $\mathbb{D}^{\prime}(X)$ and $\mathcal{E}^{\prime}(X)$ then consist of the distributions on $X$ and the distributions of compact support, respectively.

3. Solvability. For the solvability question A we consider first one of the best known class of coset spaces, the symmetric spaces $X=G / K$ of the noncompact type ( $G$ a connected semisimple Lie group with finite center, $K$ a maximal compact subgroup). Here we have for each $D \in \mathbf{D}(G / K)$ the global solvability

$$
D C^{\infty}(X)=C^{\infty}(X)
$$

as was proved in [10e] using the Fourier transform on $X$. We recall the original definition of the Fourier transform on $X$ from [10c] since we shall state some new results for it below. The Fourier transform of a function $F(y)$ on $\mathbf{R}^{n}$ can be written

$$
\tilde{F}(\eta \omega)=\int_{\mathbf{R}^{n}} F(y) e^{-i \eta(y, \omega)} d y
$$

where $\eta \geqslant 0,|\omega|=1$ and $(y, \omega)$ is the usual inner product. Geometrically, $(y, \omega)$ is the (signed) distance from the origin to the hyperplane through $y$ perpendicular to $\omega$. It turns out to have an analogue for the symmetric space $X=G / K$. To define it let $G=K A N$ be an Iwasawa decomposition of the group $G$; here $A$ is an abelian subgroup and $N$ a nilpotent subgroup. (In the case $G=\mathbf{S L}(n, \mathbf{R}), K=\mathbf{S O}(n)$ the group $A$ is the group of positive diagonal matrices with determinant $1, N$ the set of supertriangular matrices with diagonal 1; here the decomposition amounts to the usual Gram-Schmidt orthonormalization process.) Let $M$ denote the centralizer of $A$ in $K$ and $B$ the coset space $K / M$.

A horocycle in $X$ is an orbit in $X$ of a group of the form $\mathrm{gNg}^{-1}$. The group $G$ permutes the horocycles transitively. More precisely let $o=\{K\}$ (the origin in $X$ ) let $\xi_{0}$ denote the horocycle $N \cdot o$. Then each horocycle $\xi$ can be written $\xi=k a \cdot \xi_{o}$, where $k M$ is unique in $B$ (called the normal to the horocycle), and $a \in A$ is unique (called the complex distance from $o$ to $\xi$ ). This representation of $\xi$ is obvious in the case of the Poincare disk where the horocycles are the circles tangential to the unit circle. Given $x \in X, b \in B$ there is a unique horocycle through $x$ with normal $b$; let $a(x, b)$ denote its complex distance from $o$ and let $A(x, b)$ be the element in the Lie algebra a of $A$ satisfying exp $A(x, b)=a(x, b)$. This vector-valued function $A(x, b)$ on $X \times B$ is the desired analog of the scalar product $(y, \omega)$ for $\mathbf{R}^{n} \times \mathbf{S}^{n-1}$. 
The space $X$ has a certain canonical Riemannian metric and we let $d x$ denote the volume element on $X$ with respect to this metric. Let $a^{*}$ denote the dual of the vector space a.

Given a function $f(x)$ on $X$ we define its Fourier transform in analogy with (2) by

$$
\tilde{f}(\lambda, b)=\int_{X} f(x) e^{(-i \lambda+\rho)(A(x, b))} d x \quad\left(\lambda \in \mathrm{a}^{*}, b \in B\right) .
$$

Here $\rho$ is a certain fixed element of $a^{*}: 2 \rho(H)$ is the Jacobian of the automorphism $n \rightarrow \exp (H) n \exp (-H)$ of $N$. It turns out that the transform (3) is one-to-one on $\mathscr{D}(X)$. There is an inversion formula of the same type as well as a Plancherel formula relating the $L^{2}$ norms of $f$ and $\tilde{f}$. But for the proof of (1) the important result to have is a theorem of Paley-Wiener type, that is, an intrinsic characterization of the space $\mathscr{D}(X)^{\sim}$. To describe it let $W$ denote the Weyl group of $a$, that is, the (finite) group of linear transformations of $a$ induced by those elements of $K$ which normalize a. Then we have the following result [10e].

THEOREM 3.1. The space $\mathscr{D}(X)^{\sim}$ of Fourier transforms consists of the smooth functions $\phi(\lambda, b)$ on $\mathfrak{a}^{*} \times B$ satisfying:

(i) $\lambda \rightarrow \phi(\lambda, b)$ extends to an entire function of exponential type on the complexification $\mathfrak{a}_{c}^{*}$, the exponential type being uniform in $b \in B$.

(ii) For each $s \in W$ and each $\lambda \in a^{*}$,

$$
\int_{B} e^{(i s \lambda+\rho)(A(x, b))} \phi(s \lambda, b) d b=\int_{B} e^{(i \lambda+\rho)(A(x, b))} \phi(\lambda, b) d b,
$$

where $d b$ is a $K$-invariant measure on $B$.

The application of the Fourier transform (3) to prove (1) is based on the fact that the kernel in (3) is an eigenfunction of $D$, i.e.,

$$
D_{x}\left(e^{(i \lambda+\rho)(A(x, b))}\right)=P_{D}(\lambda) e^{(i \lambda+\rho)(A(x, b))},
$$

where the eigenvalue is a polynomial $P_{D}(\lambda)$, independent of $b$. After proving first local solvability of $D$ and using functional analysis tools familiar from the constant coefficient theory, (1) is reduced to proving the implication,

$$
f \in \mathscr{Q}(X), \quad \operatorname{supp}(D f) \subset V \Rightarrow \operatorname{supp}(f) \subset V
$$

tor any closed ball $V$ in $X$, supp denoting support. But using Theorem 3.1, statement (5) is translated into the following known property for holomorphic functions. If $F$ is an entire function of exponential type, $P \neq 0$ a polynomial and $P F$ of exponential type $\leqslant R$, then $F$ is of exponential type $\leqslant R$.

In order to have an analog of (1) for distributions it would be sufficient to have a topological version of Theorem 3.1. We will now describe a special result of this type. For this let $\delta$ be an irreducible unitary representation of $K$ on a vector space $V_{\delta}$ of dimension $d(\delta)$, and let $\delta^{\prime}$ denote the contragredient representation. Let $V_{\delta}^{M}$ denote the set of vectors $v \in V_{\delta}$ fixed under $\delta(M)$ and $l(\delta)=\operatorname{dim} V_{\delta}^{M}$. We assume $l(\delta)>0$. Let $\mathfrak{p}$ be the orthogonal complement of $\mathfrak{f}$ in $g$ with respect to the Killing form and $\mathfrak{p}_{c}, \mathfrak{f}_{c}$ and $\mathfrak{g}_{c}$ their respective complexifications. Let $H$ be the set of $K$-harmonic polynomials in the symmetric algebra $S\left(\mathfrak{p}_{c}\right)$ and put $H^{*}=\lambda(H)$, where $\lambda$ is the canonical 
symmetrization map of the symmetric algebra over $\mathfrak{g}_{c}$ onto the complex universal enveloping algebra $U(\mathfrak{g})$. Let $\left(v_{i}\right)$ be a basis of $V_{\delta}^{M}$ and $\left(\varepsilon_{j}\right)$ a basis of the space of linear maps of $V_{\delta}$ into $H^{*}$ commuting with the action of $K$. Since $U(\mathfrak{g})=(U(\mathfrak{g}) \mathfrak{f}+\mathfrak{n} U(\mathfrak{g})) \oplus U(\mathfrak{a})$ (where $\mathfrak{n}$ is the Lie algebra of $N$ ) we have a mapping $u \rightarrow q^{u}$ of $U(\mathrm{~g})$ into $U(\mathfrak{a})$ given by $u-q^{u} \in U(\mathrm{~g})^{\mathfrak{f}}+$ $\mathfrak{n} U(\mathrm{~g})$. As in Kostant [22a] we consider for $\lambda \in \mathrm{a}_{c}^{*}$ the complex $l(\delta) \times l(\delta)$ matrix

$$
Q^{\delta}(\lambda)=\left[q^{\varepsilon_{j}\left(v_{i}\right)}(\rho-i \lambda)\right]_{1<i, j<l(\delta)} .
$$

The space $\mathcal{H}\left(a^{*}\right)$ of entire functions of exponential type on $a_{c}^{*}$ has a natural topology with respect to which the Euclidean Fourier transform

$$
F(a) \rightarrow F^{*}(\lambda)=\int_{A} F(a) e^{-i \lambda(\log a)} d a
$$

is a homeomorphism of $\mathscr{O D}(A)$ onto $\mathcal{H}\left(\mathrm{a}^{*}\right)$ [6b]. The same holds for the space $\mathscr{H}\left(a^{*}, V\right)$ of holomorphic functions of exponential type with values in a finite-dimensional vector space $V$. Consider now the subspace

$$
\mathscr{H}^{\delta}\left(\mathfrak{a}^{*}\right)=\left\{F \in \mathscr{H}\left(\mathfrak{a}^{*}, \operatorname{Hom}\left(V_{\delta}, V_{\delta}\right)\right) \mid\left(Q^{\delta^{\prime}}\right)^{-1} F W \text {-invariant }\right\}
$$

with the relative topology, and the closed subspace of $\delta_{\delta^{\prime}}(X) \subset$ 이 $(X)$ consisting of those functions in $\mathscr{D}(X)$ which are $K$-finite of type $\delta^{\prime}$. Finally let $\Phi_{\lambda, \delta}$ denote the generalized spherical function

$$
\Phi_{\lambda, \delta}(x)=\int_{K} e^{(i \lambda+\rho)(A(x, k M))} \delta(k) d k .
$$

Then we have the following refinement of Theorem 3.1 (cf. [10g]).

THEOREM 3.2. The mapping $f \rightarrow \tilde{f}$ where

$$
\tilde{f}(\lambda)=d(\delta) \int_{X} f(x) \Phi_{\bar{\lambda}, \delta}(x)^{*} d x \quad(*=\text { adjoint })
$$

is a homeomorphism of $\mathscr{D D}_{\delta^{\prime}}(X)$ onto $\mathrm{K}^{\gamma}\left(\mathrm{a}^{*}\right)$.

In addition to Theorem 3.1, the proof uses the relationship of $Q^{\delta}(\lambda)$ to the intertwining operators for the principal series found by Johnson and Wallach [17]. From Theorem 3.2 we have immediately the following consequence.

Corollary 3.3. Let $D \neq 0$ in $\mathbf{D}(G / K)$ and let ${ }^{\prime} \mathrm{R}_{0}^{\prime}(X)$ denote the space of $K$-finite distributions on $X$. Then

$$
D \text { O }_{0}^{\prime}(X)=\text { on }_{0}^{\prime}(X) \text {. }
$$

For the space $\varepsilon^{\prime}(X)$ the analog to (7), of course, fails; as a consequence of Theorem 3.1 the range $D \varepsilon^{\prime}$ consists of those distributions $T \in \mathcal{E}^{\prime}(X)$ whose Fourier transform $\tilde{T}(\lambda, b)$ is divisible by the polynomial $P_{D}(\lambda)$ in (4) (cf. [10e, §8]).

Next let us consider the solvability question for a Lie group $L$ viewed as a homogeneous space under left translations. The operator $\partial / \partial x+i \partial / \partial y+$ 
$i x \partial / \partial z$ on $\mathbf{R}^{3}$ is a left invariant differential operator on the Heisenberg group

$$
\left[\begin{array}{lll}
1 & x & z \\
0 & 1 & y \\
0 & 0 & 1
\end{array}\right), \quad x, y, z \in \mathbf{R},
$$

yet it is essentially the same as H. Levy's operator which he proved was not locally solvable (cf. [3]). Thus the operators in $\mathbf{D}(L)$ are not, in general, locally solvable.

The contrast between this negative result for $L$ and the positive results for the symmetric space $G / K$ disappears when we view $L$ as a symmetric space. A coset space $B / C$ ( $B$ a Lie group, $C$ a closed subgroup) is called a symmetric coset space if there exists an involutive automorphism $\sigma$ of $B$ with fixed point set $C$. The spaces $G / K$ considered above have this property. Now we consider $L$ as a homogeneous space under left and right translations simultaneously, i.e., we let the product group $L \times L$ act on $L$ by

$$
\left(g_{1}, g_{2}\right): g \rightarrow g_{1} g g_{2}^{-1}, \quad g \in L .
$$

The subgroup leaving $e$ fixed is the diagonal $L^{*} \cong L$ so we have the coset space representation

$$
L=(L \times L) / L^{*} .
$$

Then the algebra $\mathbf{D}\left(L \times L / L^{*}\right)$ is canonically isomorphic to the algebra $\mathbf{Z}(L)$ of bi-invariant differential operators on $L$, and the natural problem becomes: Given $D \in \mathbf{Z}(L)$, is it globally (respectively, locally) solvable? In [5], Duflo and Raïs proved

THEOREM 3.4. Let $L$ be a solvable Lie group. Then each bi-invariant differential operator $D \neq 0$ is locally solvable.

This had been proved in Raïs [31] for the special case of a nilpotent Lie group. Their proof is based on a detailed description of the operators $D \in \mathbf{Z}(L)$ by means of harmonic analysis on $L$, whereby a local fundamental solution can be constructed, giving local solvability. An entirely different proof was given recently by $\mathrm{F}$. Rouvière [33]. He proves that given $D \in \mathbf{Z}(L)$ there exists a bi-invariant differential operator $E \neq 0$ on the derived group $L^{\prime}$ such that for $L^{2}$ norms on $L$,

$$
\|E u\| \leqslant\|D u\|
$$

for $u \in C^{\infty}(L)$ of sufficiently small support. On the other hand Hörmander showed [15, p. 157] that local solvability of $E$ on $L^{\prime}$ implies certain sup norm inequalities which, using the invariance of $E$, Rouvière converts into norm inequalities,

$$
\left|\int_{L^{\prime}} u(x) v(x) d x\right| \leqslant C\|u\|_{k}\left\|^{t} E v\right\|_{l^{\prime}} .
$$

Here $d x$ is a right invariant Haar measure, $C$ a constant, ${ }^{t} E$ the transpose of $E,\|\|_{k}$ a Sobolev-type norm (involving $L^{2}$ norm of derivatives up to the $k$ th order) and $u$ and $v$ have sufficiently small support. On the other hand it is well known (cf. [15, p. 178] or [38, p. 142]) that (10) implies local solvability of $E$ on $L^{\prime}$. Now (9) and (10) imply by elementary estimates that (10) holds for 
the operator $D$ on $L$. Thus local solvability of $E$ implies that of $D$ so the theorem follows by induction. ${ }^{5}$

THEOREM 3.5. Let $L$ be a semisimple Lie group. Then each bi-invariant differential operator $D \neq 0$ is locally solvable.

This is proved in [10e] using Lemma 24 in [8a] which relates explicitly the action of $D$ on invariant distributions with the action of a constant coefficient operator on the Lie algebra, together with a result of [31] that all invariance properties of a constant coefficient operator are preserved in a suitable fundamental solution.

Global solvability does not, in general, hold in Theorem 3.5, as shown by the following interesting example due to Cerèzo and Rouvière [3]. Let $G$ and $K$ be as above and assume that $\mathrm{g}$ has a complex structure $J$; then $\mathrm{g}=\mathfrak{f}+J \mathfrak{f}$. Let $\left(T_{i}\right)$ be a basis of $\mathfrak{f}$, orthonormal with respect to the negative of the Killing form on $\mathrm{g}$. Then the operator $\omega=\Sigma_{i}\left(J T_{i}\right) T_{i}$ lies in $\mathbf{Z}(G)$ but annihilates all functions on $G$ which are right invariant under $K$, hence is not globally solvable.

Nevertheless we have the global solvability

$$
\Omega C^{\infty}(G)=C^{\infty}(G)
$$

for the Casimir operator $\Omega$ on any connected noncompact semisimple Lie group $G$. This was proved in [3] for complex $G$ using harmonic analysis on $G$. For general semisimple $G$, (11) was proved by Rauch and Wigner [32]. The main ingredient in their proof is the verification that no null bicharacteristics of $\Omega$ lie over a compact subset of $G$. They also prove that $\Omega$ is injective on OD $(G)$ and has a property similar to (4). Some general results of that nature have been proved by K. Johnson [16].

Since a general Lie group $L$ decomposes into a semisimple Lie group and a solvable Lie group (strict semidirect product if the big group is simply connected) one could hope for a joint generalization of Theorems 3.4 and 3.5 to all Lie groups. ${ }^{6}$ But taking (1) into account as well as the representation (8) of $L$ as a symmetric coset space, we are led to an even more general question:

Let $B / C$ be a symmetric coset space and $D$ a $B$-invariant differential operator on it. Is D necessarily locally solvable?

4. Joint eigenfunctions. We shall now give a survey of relatively recent work towards determining the joint eigenfunctions of the operators in $\mathbf{D}(G / K)$. First we consider the case when $G / K$ is a symmetric space $X$ of the noncompact type. The joint eigenfunctions can be characterized (cf. [10b, p. 439]) by the functional equation

$$
\phi(o) \int_{K} \phi(g k \cdot x) d k=\phi(g \cdot o) \int_{K} \phi(k \cdot x) d k,
$$

5 After this was written I received a preprint [4] from M. Duflo establishing this local solvability in general. His proof combines new algebraic results about $\mathbf{Z}(L)$ with the method of Theorem 3.4.

${ }^{6}$ ADDED IN PROOF. In a recent preprint [42], Wigner proves global solvability for a simply connected nilpotent Lie group. 
$g \in G, x \in G / K$. By (4) in $\S 3$ the functions $x \rightarrow e^{\nu(A(x, b))}\left(\nu \in \mathrm{a}_{c}^{*}\right)$ are joint eigenfunctions and the inversion formula for the Fourier transform (3) suggests them as building blocks for all joint eigenfunctions. HarishChandra's integral formula

$$
\psi_{\nu}(x)=\int_{B} e^{\nu(A(x, b))} d b \quad\left(\nu \in \mathfrak{a}_{c}^{*}\right)
$$

for the $K$-invariant eigenfunctions on $X$ (the spherical functions) is the prototype of the integral representations one might expect for the solutions of (1). In Furstenberg [7b] and Karpelevič [18] the following result is proved.

THEOREM 4.1. The functions

$$
x \rightarrow \int_{B} e^{\nu(A(x, b))} d \mu(b), \quad x \in X,
$$

where $\nu \in \mathfrak{a}^{*}$ and $\mu$ a positive measure on $B$ constitute all the positive joint eigenfunctions of $\mathbf{D}(G / K)$.

Furstenberg's proof is purely measure-theoretic. It consists of proving, using methods of Choquet and Deny, that the convex cone of positive solutions to (1) is generated by the extremal rays, which then are related to the integrand in (3). Karpelevič's method goes further and he gives an integral representation of the positive eigenfunctions of the Laplace-Beltrami operator $L$ on $G / K$. A fundamental solution for the operator $L$-const is constructed rather explicitly via the heat equation on $G / K$ and the minimal solutions are constructed from the fundamental solution by means of the method of Martin [26]. Since any positive eigenfunction is a measure-theoretic superposition of minimal eigenfunctions, the integral representation for positive eigengenfunctions of $L$ follows; formula (3) is a simple corollary.

The problem of determining all joint eigenfunctions was raised in [10d, $p$. 139] and it was proved for the hyperbolic disk that analytic functionals (alias hyperfunctions) on the boundary are what is needed to give (by superposition) all the eigenfunctions of $L$.

THEOREM 4.2. The eigenfunctions of the Laplace-Beltrami operator on the hyperbolic space $X$ are precisely the functions

$$
x \rightarrow \int_{B} e^{\nu(A(x, b))} d T(b)
$$

where $\nu \in a_{c}^{*}$ and $T$ an analytic functional on $B$.

The method amounts to first determining the $K$-finite eigenfunctions of $L$ in terms of hypergeometric functions, and then showing, using asymptotic properties of the hypergeometric functions, that the convergence of the expansion of an arbitrary eigenfunction on $X$ into $K$-finite ones implies convergence of the boundary values in the sense of analytic functionals. This method gives the following partial solution for $X$ of rank one.

THEOREM 4.3. Let $X$ be symmetric of rank one. The functions (4) for $\nu$ real (i.e., $\nu \in \mathfrak{a}^{*}$ ) constitute all the eigenfunctions of $L$ with eigenvalue $\geqslant-\langle\rho, \rho\rangle$. 
For the proof see [10f]. Intermediary results extending Theorem 4.2, using a similar method, were given by Hashizume, Minemura and Okamoto (cf. [9b], $[27 \mathrm{a}, \mathbf{b}, \mathbf{c}])$.

The $K$-finite joint eigenfunctions of $\mathbf{D}(G / K)$ are determined in [10d, $\mathbf{g}]$ as follows, generalizing (2).

THEOREM 4.4. Let $G / K$ be arbitrary. The $K$-finite joint eigenfunctions of $\mathbf{D}(G / K)$ are precisely the functions

$$
\Phi_{\nu}(x)=\int_{B} e^{\nu(A(x, b))} F(b) d b
$$

where $\nu \in a_{c}^{*}$ and $F$ is a $K$-finite function on $B$. Moreover, there exists a right invariant differential operator $D$ on $G$ such that

$$
\Phi_{\nu}(g K)=D_{g}\left(\psi_{\nu}(g K)\right), \quad g \in G .
$$

The proof is primarily based on the Paley-Wiener theorem for $K$-finite functions (Theorem 3.2). As pointed out in [10d, p. 138] for $X$ of rank one, Theorem 4.4 implies that each joint eigenfunction of $\mathbf{D}(G / K)$ has the form

$$
f(x)=\int_{B} e^{\nu(A(x, b))} d T(b),
$$

where $T$ is a "functional" on $B$ (possibly depending on $\nu$ and $f$ ). The conjecture, suggested by Theorems $4.2-4.3$, that these functionals are just the analytic functionals (hyperfunctions) on $B$ was taken up in a remarkable cooperation of Kashiwara, Kowata, Minemura, Okamoto, Oshima, and Tanaka (cf. [19]), and they proved the following general result.

THEOREM 4.5. Assume $\lambda \in \mathfrak{a}_{c}^{*}$ satisfies $^{7}$

$$
2\langle i \lambda, \alpha\rangle /\langle\alpha, \alpha\rangle \notin \mathbf{Z}
$$

for all restricted roots $\alpha$ of $\mathfrak{g}$ with respect to $a$ and let $\mu: \mathrm{D}(G / K) \rightarrow \mathbf{C}$ be the homomorphism given by $\mu(D)=P_{D}(\lambda)$ in the notation of $(4), \S 3$. Then the functions

$$
f(x)=\int_{B} e^{(i \lambda+\rho)(A(x, b))} d T(b),
$$

where $T$ is a hyperfunction on $B$ constitute all the elements in the joint eigenspace $E_{\mu}$.

The proof uses many results from the extensive theory of hyperfunctions while Theorem 4.3 only requires a characterization of their spherical harmonics expansion.

It is of interest to observe that if $X$ has rank 1 and if condition (6) fails, the condition of Theorem 4.3 is satisfied, so we have

THEOREM 4.6. If $X$ has rank one the functions (7) constitute all the eigenfunctions of the Laplacian on $X$.

In his paper [24], J. Lewis investigates the functions (7) as $T$ runs through the space ${ }^{\prime} D^{\prime}(B)$ of distributions on $B$, and proves the following result.

\footnotetext{
${ }^{7}$ ADDED IN PROOF. In a recent communication Minemura and Oshima avoid this restriction.
} 
THEOREM 4.7. If $T \in \mathcal{O D}^{\prime}(B)$ then the function $f(x)$ in (7) grows no faster than $e^{C d(0, x)}(C=$ constant, $d$ distance). The converse holds for $X$ of rank one provided (6) is satisfied.

In the case when $G / K$ is a Hermitian symmetric space there are integral representations of the type (3) (with $B$ replaced by the Bergman-Silov boundary) for bounded holomorphic functions [13], [25], [7a], [28], and for solutions of a certain overdetermined system [21].

We can also consider the case when $G / K=\mathbf{R}^{n}(n>1)$ and $G$ the group of all isometries. Here $\mathbf{D}(G / K)$ is generated by the Laplacian $L$; from general results of Ehrenpreis [6a] we have that the solutions to $L u=-\lambda^{2} u(\lambda \in \mathbf{C})$ are given by

$$
u(x)=\int e^{i(x, \zeta)} d \mu(\zeta)
$$

where $\mu$ is a measure with support on $\left\{\zeta \in \mathbf{C}^{n} \mid \zeta_{1}^{2}+\cdots+\zeta_{n}^{2}=\lambda^{2}\right\}$ satisfying

$$
\int e^{-(x, \operatorname{Im} \xi)}(1+|\zeta|)^{N} d \mu(\zeta)<\infty
$$

for all $N$ and all $x \in \mathbf{R}^{n}$; here, however, $\mu$ is not unique. One can also prove (for $\lambda \neq 0$ ) an integral formula

$$
u(x)=\int_{\mathbf{S}^{n-1}} e^{i \lambda(x, \omega)} d T(\omega)
$$

for the solutions to $L u=-\lambda^{2} u$ in analogy with (4) (cf. [9a], [10f]), but then $T$ is more general than an analytic functional: for $n=2$ the $T$ are the continuous linear functionals on the space of analytic functions on $\mathbf{S}^{\mathbf{1}}$ which extend to holomorphic functions $f$ on $\mathbf{C}-(0)$ such that $f(z)$ and $f\left(z^{-1}\right)$ have exponential type at $z=\infty$ (cf. [10f]).

Next we consider the case of a simply connected symmetric space $U / K$ of the compact type. We assume $U / K$ dual to the noncompact space $G / K$ in Theorem 4.4, that is, $G$ is a subgroup of the simply connected complexification $G_{c}$, whose Lie algebra $\mathfrak{g}_{c}$ is the complexification of $\mathrm{g}$ as well as of the Lie algebra $u$ of $U$. We also take $U$ to be the analytic subgroup of $G_{c}$ with Lie algebra $u$; then $U$ and $U / K$ are both simply connected.

Let $A(g)=A(g K, e M)(g \in G)$ so $g=n \exp A(g) k$ with $n \in N, k \in K$. The mapping $A: G \rightarrow$ a can be extended to a holomorphic mapping $A$ : $G_{c}^{0} \rightarrow \mathfrak{a}_{c}$ of a neighborhood $G_{c}^{0}$ of the identity in $G_{c}$ into the complexification $\mathfrak{a}_{c}$ of $\mathfrak{a}$ as follows (Stanton [36], Sherman [35b]). For the complexified Iwasawa decomposition $\mathfrak{g}_{c}=\mathfrak{n}_{c}+\mathfrak{a}_{c}+\mathfrak{f}_{c}$ the mapping

$$
(X, H, T) \rightarrow \exp X \exp H \exp T
$$

is a holomorphic diffeomorphism of a neighborhood of $(0,0,0)$ in $\mathfrak{n}_{c} \times \mathfrak{a}_{c} \times$ $\mathfrak{f}_{c}$ onto a neighborhood $G_{c}^{0}$ of $e$ in $G_{c}$. The mapping

$$
\exp X \exp H \exp T \rightarrow H
$$

is then the desired holomorpnic map $A: G_{c}^{0} \rightarrow \mathfrak{a}_{c}$. Taking $\mathfrak{a}_{c}$ with the customary Hilbert space inner product $(X, Y) \rightarrow-B(X, \tau Y)$, where $\tau$ is the conjugation of $\mathfrak{g}_{c}$ with respect to $\mathfrak{u}$, we may take $G_{c}^{0}$ as the diffeomorphic image (under exp) of an open ball $B_{0} \subset \mathfrak{g}_{c}$ with center 0 . Let 


$$
U_{0}=\exp \left(B_{0} \cap \mathfrak{u}\right) \text {. }
$$

It is clear that for each $\nu \in \mathrm{a}_{c}^{*}$ the function $u \rightarrow e^{\nu(A(u))}$ on $U_{0}$ is an eigenfunction of each $D \in \mathbf{D}(U / K)$; moreover, as noted in Stanton [36] and Sherman [35a, b], the spherical functions on $U / K$ are (by analytic continuation) given by the analog of Harish-Chandra's formula (2), namely

$$
\phi(u K)=\int_{K} e^{-\mu\left(A\left(k u k^{-1}\right)\right)} d k, \quad u \in U_{0}
$$

where $\mu$ is the highest weight of the spherical representation associated with $\phi$. More generally we have (cf. [10h])

THEOREM 4.8. Each joint eigenfunction of $\mathbf{D}(U / K)$ has the form

$$
f(u K)=\int_{K / M} e^{-\mu\left(A\left(k^{-1} u k\right)\right)} F(k M) d k_{M} \quad\left(u \in U_{0}\right)
$$

where $d k_{M}=d b, F \in C^{\infty}(K / M)$ and $\mu \in \mathfrak{a}^{*}$ satisfies $\langle\mu, \alpha\rangle /\langle\alpha, \alpha\rangle \in \mathbf{Z}^{+}$ for all restricted roots $\alpha>0$. Conversely, if $\mu$ satisfies this condition the function $f$ extends uniquely to an analytic function on $U / K$ which is a joint eigenfunction of $\mathbf{D}(U / K)$.

Next we consider for a symmetric space $G / K$ of the noncompact type the space $\Xi=G / M N(\S 3)$, which is identified with the space of horocycles in $G / K$, and has many properties analogous to those of $G / K$. In particular, the $G$-invariant differential operators on $\Xi$ are all of the form

$$
\begin{aligned}
& \left(D_{P} \phi\right)(g M N) \\
& \quad=\left\{P\left(\frac{\partial}{\partial t_{1}}, \ldots, \frac{\partial}{\partial t_{l}}\right) \phi\left(g \exp \left(t_{1} H_{1}+\cdots+t_{l} H_{l}\right) M N\right)\right\}_{t=0}
\end{aligned}
$$

where $P \in S\left(a_{c}\right)$ and the mapping $P \rightarrow D_{P}$ is an isomorphism of $S\left(a_{c}\right)$ onto $\mathbf{D}(G / M N)$ [10d]. Thus the solvability questions of $\$ 3$ reduce to constant coefficient questions. Noting that $G / M N=(K / M) \times A$ we find easily the following answer to Problem B, $\$ 2$.

Proposition 4.9. The joint eigendistributions of $\mathbf{D}(G / M N)$ are given by

$$
\Psi(\phi)=\int_{K / M} \int_{A} \phi(k a M N) e^{(i \lambda+\rho)(\log a)} d a d S(k M) \quad(\phi \in \text { ol }(\Xi))
$$

where $\lambda \in \mathfrak{a}_{c}^{*}$ and $S \in \mathrm{v}^{\prime}{ }^{\prime}(B)$. The eigenvalues are polynomials in $\lambda$, independent of $S$.

Let $\operatorname{~ID~}_{\lambda}^{\prime}(\Xi)$ denote the space of the distributions $\Psi$ in (9). Of particular interest are the analogs of the spherical functions on $G / K$, namely the $M N$-invariant distributions $\Psi$ in (9), the so-called conical distributions. In contrast to the situation for spherical functions it was natural to conjecture that the set of conical distributions in $"{ }^{2}{ }_{\lambda}^{\prime}$ would be parametrized by $W$. Theorems 4.10, 4.12 give a partial confirmation of this. In order to determine these distributions we decompose $\Xi$ according to the Bruhat decomposition of $G$. For each $s \in W$ fix $m_{s} \in K$ such that $\operatorname{Ad}\left(m_{s}\right) \mid \mathfrak{a}=s$. Then if $\xi_{s}=$ $m_{s} M N, \Xi_{s}=M N A \cdot \xi_{s}$, we have $\Xi=\cup_{s \in W} \Xi_{s}$ (disjoint union). If $\xi \in \Xi_{s}$ we write $a(\xi)$ for the (unique) $A$ factor in $\xi=m n a(\xi) \cdot \xi_{s}$. Let $\Sigma^{+}$(respectively 
$\left.\Sigma^{-}\right)$denote the set of positive (negative) roots of $\mathfrak{g}$ with respect to $\mathfrak{a}, \Sigma_{0}^{+}\left(\Sigma_{0}^{-}\right)$ the corresponding sets of reduced roots. If

$$
\operatorname{Re}\langle i \lambda, \alpha\rangle>0 \text { for } \alpha \in \Sigma^{+} \cap s^{-1} \Sigma^{-},
$$

then the function $\xi \rightarrow e^{(i s \lambda+s \rho)(\log a(\xi))}$ on $\Xi_{s}$, viewed as a distribution on $\Xi$, is a conical distribution $\Psi_{\lambda, s}^{\prime} \in \mathscr{D}_{\lambda}^{\prime}$. Moreover, if $\Gamma$ is the Gamma function and we put $\alpha_{0}=\alpha /\langle\alpha, \alpha\rangle$, the mapping

$$
\lambda \rightarrow \Psi_{\lambda, s}=\left(\prod_{\alpha \in \Sigma_{0}^{+} \cap s^{-1} \Sigma^{-}} \Gamma\left(\left\langle i \lambda, \alpha_{0}\right\rangle\right)\right)^{-1} \Psi_{\lambda, s}^{\prime}
$$

extends to a holomorphic function on $\mathfrak{a}_{c}^{*}$ with values in $\left.{ }^{D}\right)^{\prime}(\Xi)$ and each $\Psi_{\lambda, s}$ is a conical distribution in ${ }^{D_{\lambda}^{\prime}}(\Xi)[\mathbf{1 0 d}$, p. 88]. Let

$$
\pi(\lambda)=\prod_{\alpha \in \Sigma_{0}^{+}}\langle\lambda, \alpha\rangle
$$

and let $\mathbf{e}(\lambda)^{-1}$ denote the denominator in Harish-Chandra's c-function.

(11) $\mathbf{e}(\lambda)^{-1}=\prod_{\alpha \in \Sigma_{0}^{+}} \Gamma\left(\frac{1}{2}\left(\frac{1}{2} m_{\alpha}+1+\left\langle i \lambda, \alpha_{0}\right\rangle\right)\right) \Gamma\left(\frac{1}{2}\left(\frac{1}{2} m_{\alpha}+m_{2 \alpha}+\left\langle i \lambda, \alpha_{0}\right\rangle\right)\right)$, $m_{\alpha}$ and $m_{2 \alpha}$ denoting the respective multiplicities. Then we have, by [10d, p. 96] and [10g, Theorem 6.1],

THEOREM 4.10. Let $\lambda \in \mathfrak{a}_{c}^{*}$ and assume

$$
\pi(\lambda) \mathbf{e}(\lambda) \neq 0 \text {. }
$$

Then the linear combinations $\Sigma_{s \in W} c_{s} \Psi_{\lambda, s}, c_{s} \in \mathbf{C}$, constitute all the conical distributions in $\mathscr{D}_{\lambda}^{\prime}(\bar{\Xi})$.

Of the orbits $\Xi_{s}$ above, exactly one, say $\Xi_{s^{*}}$, is an open subset of $\Xi$, the others have lower dimension. For the corresponding distribution $\Psi_{\lambda, s^{*}}$ we have

THEOREM 4.11. The distribution $\Psi_{\lambda, s^{*}}$ is given by a locally integrable function on $\Xi$ if and only if

$$
\operatorname{Re}(\langle i \lambda, \alpha\rangle)>0 \quad \text { for } \alpha \in \Sigma^{+},
$$

and by $a C^{\infty}$ function on $\Xi$ if and only if

$$
\left\langle i \lambda-\rho, \alpha_{0}\right\rangle \in \mathbf{Z}^{+} \text {for } \alpha \in \Sigma^{+} \text {. }
$$

For $X$ of rank one, $\mathrm{Hu}[\mathbf{1 4 a}, \mathbf{b}]$ managed to eliminate the discrete set of $\lambda$ which violates condition (12). Since all $\Psi_{0, s}(s \in W)$ are proportional, an additional conical distribution $\Psi_{0} \in \mathscr{O}_{0}^{\prime}$ is needed. It is defined as follows. The function $e^{\rho(\log a(\xi))}$ on the open dense subset $\Xi_{s^{*}}$ is not locally integrable on $\Xi$, but the following regularization

$$
\Psi_{0}(\phi)=\int_{\Xi}\left(\phi(\xi)-\phi_{0}(\xi)\right) e^{\rho(\log a(\xi))} d \xi .
$$

where for $\phi \in \cup D(\Xi), \phi_{0}$ is given by $\phi_{0}(k a M N)=\phi(a M N)$, gives a welldefined conical distribution in ${ }^{2} D_{0}^{\prime}$. Defining conical distributions by means of the full isometry group $\tilde{G}$ of $X$ instead of $G$ (this makes a difference only for $G=\operatorname{SL}(2, \mathbf{R}))$, Hu proved the following result. 
THEOREM 4.12. If $X$ has rank one the linear combinations $c \Psi_{\lambda, s^{*}}+c^{\prime} \Psi_{\lambda, e}$ $\left(c \Psi_{0}+c^{\prime} \Psi_{0, e}\right.$ for $\left.\lambda=0\right)$ constitute all the conical distributions in $\mathscr{D Q D}_{\lambda}^{\prime}(\Xi)$.

At the poles of $\Psi_{\lambda, s^{*}}^{\prime}$ the residues are conical distributions which are certain transversal derivatives of $\Psi_{\lambda, e}$. For an algebraic approach giving part of Theorem 4.12 see Lepowsky [23]. In view of Theorems 4.5-4.6 and the fact that the dual Radon transform maps joint eigenspaces of $\mathbf{D}(G / M N)$ into joint eigenspaces of $\mathbf{D}(G / K)$ [10d, p. 93] it would be natural to consider the joint eigenhyperfunctions of $\mathbf{D}(G / M N)$. While the necessary adjustment in Theorem 4.9 is obvious, one might wonder whether a conical hyperfunction is not necessarily a distribution?

5. Eigenspace representations. In this section we state without proof some results on the eigenspace representations for certain classes of homogeneous spaces $G / K$ (Problem $C$ in $\$ 2$ ). Again we start with the case when $G / K$ is a symmetric space of the noncompact type. Since each joint eigenspace contains a spherical function $((2), \S 4)$ it is clear that each joint eigenspace has the form

$$
\varepsilon_{\lambda}(X)=\left\{f \in C^{\infty}(X) \mid D f=P_{D}(\lambda) f \text { for } D \in \mathbf{D}(G / K)\right\} .
$$

Here $\lambda \in a_{c}^{*}$ is arbitrary and $P_{D}$ is the polynomial in (4), §3. Let $T_{\lambda}$ denote the representation of $G$ on $\mathcal{E}_{\lambda}(X)$ given by $\left(T_{\lambda}(g) f\right)(x)=f\left(g^{-1} \cdot x\right)$. Since $P_{D}$ is $W$-invariant we have $\varepsilon_{s \lambda}=\varepsilon_{\lambda}, T_{s \lambda}=T_{\lambda}$ for all $s \in W$. Using many different tools the following result is proved in $[10 \mathrm{~g}]$.

THEOREM 5.1. The eigenspace representation $T_{\lambda}$ is irreducible if and only if

$$
\mathbf{e}(\lambda) \mathbf{e}(-\lambda) \neq 0
$$

in the notation of (11), $\S 4$.

For the non-Euclidean disk with the Riemannian metric

$$
d s^{2}=\left(1-x^{2}-y^{2}\right)^{-2}\left(d x^{2}+d y^{2}\right)
$$

the criterion (1) gives the following: The eigenspace of the Laplacian $L$ with eigenvalue $4 c(c-1)$ is irreducible if and only if $c$ is not an integer.

Next we consider the case when $G / K=\mathbf{R}^{n}(n>1)$ and $G$ is the group of all isometries. For $\lambda \in \mathbf{C}$ let $\varepsilon_{\lambda}\left(\mathbf{R}^{n}\right)$ denote the eigenspace

$$
\varepsilon_{\lambda}\left(\mathbf{R}^{n}\right)=\left\{f \in C^{\infty}\left(\mathbf{R}^{n}\right) \mid L f=-\lambda^{2} f\right\},
$$

$L$ being the Laplacian.

THEOREM 5.2 [10f]. The natural action of $G$ on $\varepsilon_{\lambda}\left(\mathbf{R}^{n}\right)$ is irreducible if and only if $\lambda \neq 0$.

For $\lambda=0$ the harmonic polynomials of degree $\leqslant k$ clearly form a closed, proper invariant subspace. But for eigenvalue 0 the eigenspace of $L$ is 
mapped into itself by a transformation $\tau$ of $\mathbf{R}^{n}$ provided $L$ is just quasi-invariant under $\tau$, i.e., satisfies $L^{\tau}=\phi L$ where $\phi$ is a function (this amounts to stability in Lie's sense $(\S 1))$. For example the conformal map

$$
\tau: z \rightarrow(a z+b)(c z+d)^{-1}
$$

of $\mathbf{R}^{2} \cup\{\infty\}$ satisfies $L^{\tau}=|c z-a|^{4} L$. More generally, Orsted [40] shows that if $X$ is a conformal vector field on $\mathbf{R}^{n}$ then the operator

$$
\eta(X) f=X f-\frac{n-2}{2 n}(\operatorname{div} X) f, \quad f \in C^{\infty}\left(\mathbf{R}^{n}\right),
$$

satisfies

$$
L \eta(X) f-\eta(X) L f=-(2 / n)(\operatorname{div} X) L f
$$

and $X \rightarrow \eta(X)$ is a representation of the Lie algebra $\tau$ of conformal vector fields on $\mathbf{R}^{n}$ on $C^{\infty}\left(\mathbf{R}^{n}\right)$. By (3) the space $\varepsilon_{0}\left(\mathbf{R}^{n}\right)$ of harmonic functions is $\eta$-invariant. We have then the following complement to Theorem 5.2 (cf. [10h]).

THEOREM 5.3. The representation $X \rightarrow \eta(X)$ of the conformal algebra $\tau$ on the space of harmonic functions is scalar irreducible (that is, the only commuting operators are the scalars).

For a coset space $U / K$ of compact groups $K$ and $U^{\prime}$ the irreducibility question for the eigenspace representations is quite easy.

Proposition 5.4. For compact Lie groups $K \subset U$ the eigenspace representations for $U / K$ are all irreducible under the action of $U$.

In fact, each joint eigenspace is finite dimensional and can be decomposed into a direct sum of $U$-invariant subspaces. Each of these contains a spherical function so the irreducibility follows from the fact that a spherical function on $U / K$ is completely determined by the eigenvalues of $\mathbf{D}(U / K)$.

Next we consider the horocycle space $G / M N$ associated with the noncompact symmetric space $G / K$. The joint eigenspaces (of distributions) are the spaces $\mathscr{Q}_{\lambda}^{\prime}(\Xi)\left(\lambda \in \mathfrak{a}_{c}^{*}\right)$ defined in $\S 4$. Let $\tau_{\lambda}$ denote the representation of $G$ on ' $2{ }_{\lambda}$. A representation $\tau$ is called conical if there is a fixed vector under $\tau(M N) ; \tau$ is called spherical if there is a fixed vector under $\tau(K)$. The following result describes the finite-dimensional subrepresentations of $\tau_{\lambda}$ (cf. [10d, p. 144]).

THEOREM 5.5. Let OD $_{\lambda, G}^{\prime}$ denote the space of $G$-finite vectors in $\left(\mathrm{L}_{\lambda}^{\prime}\right.$. Then:

(i) ${ }^{\circ}{ }_{\lambda, G}^{\prime} \neq\{0\} \Leftrightarrow\left\langle i \lambda-\rho, \alpha_{0}\right\rangle \in \mathbf{Z}^{+}$for $\alpha \in \Sigma^{+} \quad\left(\alpha_{0}=\alpha /\langle\alpha, \alpha\rangle\right)$.

(ii) The representation $\sigma_{\lambda}$ of $G$ on $(1)_{\lambda, G}^{\prime}$ is finite dimensional and irreducible.

(iii) The representations $\sigma_{\lambda}$ are precisely the finite-dimensional irreducible conical representations of $G$.

(iv) The representations $\sigma_{\lambda}$ are precisely the finite-dimensional irreducible spherical representations of $G$.

(v) The lowest weight of $\sigma_{\lambda}$ has restriction to a given by $-i \lambda+\rho$.

For the case when $G$ is complex this can be stated in a somewhat sharper form. 
THEOREM 5.6. Let $G$ be complex semisimple and simply connected and for each homomorphism $\chi: \mathbf{D}(G / N) \rightarrow \mathbf{C}$ let $\mathfrak{H}_{\chi}$ be the space of holomorphic functions $f$ on $G / N$ satisfying

$$
D f=\chi(D) f \text { for } D \in \mathbf{D}(G / N) \text {. }
$$

Let $\pi_{\chi}$ denote the representation of $G$ on $\dot{H}_{\chi}$. As $\chi$ varies, $\pi_{\chi}$ runs through all the irreducible finite-dimensional holomorphic representations of $G$.

For the eigenspace representation $\tau_{\lambda}$ of the real semisimple $G$ on $\left.{ }^{2}\right)_{\lambda}^{\prime}(\Xi)$ the following irreducibility criterion holds [22a, p. 631], [10g, §12].

THEOREM 5.7. $\tau_{\lambda}$ is irreducible if and only if $\mathbf{e}(\lambda) \mathbf{e}(-\lambda) \neq 0$.

For simply connected nilpotent Lie groups $L$ the eigenspace representations have been investigated by $A$. Hole [11]. For a linear function $\lambda \neq 0$ on the Lie algebra $\mathfrak{l}$ of $L$, let $\mathfrak{f} \subset \mathfrak{l}$ be a subalgebra of maximal dimension satisfying $\lambda([\mathfrak{f}, \mathfrak{f}])=0$ and put $\mathfrak{h}=\mathfrak{f} \cap \operatorname{kernel}(\lambda)$. Let $H \subset L$ be the analytic subgroup corresponding to $\mathfrak{h}$.

THEOREM 5.8 [11]. The eigenspace representations for $L / H$ are irreducible for all nonzero homomorphisms $\mu: \mathbf{D}(L / H) \rightarrow \mathbf{C}$. The unitary irreducible representations of $L$ (cf. [20]) can be realized in this way.

We finally indicate how the principal series of a semisimple Lie group $G$ arises from eigenspace representations on suitable vector bundles over $G / M N$ and how the discrete series arises from eigenspace representations on suitable vector bundles over $G / K$.

Let $\delta$ be an irreducible unitary representation of $M$ on $V_{\delta}$ and $\lambda \in \mathfrak{a}_{c}^{*}$. Let $\Gamma_{\delta, \lambda}$ denote the space of $C^{\infty}$ functions $f: G \rightarrow V_{\delta}$ satisfying

$$
f(\text { gman }) \equiv \delta(m)^{-1} e^{(i \lambda-\rho)(\log a)} f(g)
$$

and $\pi_{\delta, \lambda}$ the natural representation of $G$ on $\Gamma_{\delta, \lambda}$. These representations form the principal series for $G$.

Extending $\delta$ to a representation of $M N$ on $V_{\delta}$ by $\delta(m n)=\delta(m)$, we consider the associated vector bundle $E_{\delta}$ over $G / M N$. It is clear from the structure of $\mathbf{D}(G / M N)((8), \S 4)$, that the members of the principal series are the eigenspace representations of $\mathbf{D}(G / M N)$ on the section space $\Gamma\left(E_{\delta}\right)$.

In conclusion we recall that if $G$ has a compact Cartan subgroup it was proved by Takahashi [37] for the de Sitter group, by Hotta [12] and Wallach [39], in general, that the discrete series of $G$ can be realized on eigenspaces of the Casimir operator on the space of square integrable sections of vector bundles over $G / K$. It would be of interest to know the action of the bigger algebra $\mathbf{D}^{\delta}(G / K)$ on these sections as well as of the algebra $\mathbf{D}^{\delta}(G / M N)$ on $\Gamma\left(E_{\delta}\right)$ above.

Related realizations of the discrete series on nullspaces of the Dirac operator on certain vector bundles over $G / K$ were given by Parthasarathy [30] and Schmid [34]; this is extended by Wolf in [41] to the representations of $G$ which make up the Plancherel measure of $G$. 


\section{BIBLIOGRAPHY}

1. R. Bott, The index theorems for homogeneous differential operators, Differential and Combinatorial Topology (S. S. Cairns, Editor), Princeton Univ. Press, Princeton, N. J., 1965, pp. 167-186. MR 31 \#6246.

2. E. Cartan, Sur la détermination d'un système orthogonal complèt dans un espace de Riemann symétrique clos, Rend. Circ. Mat. Palermo 53 (1929), 217-252.

3. A. Cerèzo and F. Rouvière, Opérateurs différentiels invariants sur un groupe de Lie, Séminaire Goulaouic-Schwartz 1972-1973: Équations aux dérivées partielles et analyse fonctionnelle, Exposé No. 10, École Polytechnique, Paris, 1973. MR 52 \#863.

4. M. Duflo, Opérateurs differentiels bi-invariants sur un groupe de Lie (preprint).

5. M. Duflo and M. Raiis, Sur l'analyse harmonique sur les groupes de Lie résolubles, Ann. Sci. École Norm. Sup. 9 (1976), 107-144.

6a. L. Ehrenpreis, A fundamental principle for systems of linear differential equations with constant coefficients, and some of its applications, Proc. Internat. Sympos. Linear Spaces, Jerusalem, 1960, pp. 161-174. MR 24 \# A3420.

6b. __ Fourier analysis in several complex variables, Wiley-Interscience, New York, 1970. MR 44 \#3066.

7a. H. Furstenberg, A Poisson formula for semi-simple Lie groups, Ann. of Math. (2) 77 (1963), 335-386. MR 26 \#3820; 28 \# 1246.

7b. Translation-invariant cones of functions on semi-simple Lie groups, Bull. Amer. Math. Soc. 71 (1965), 271-326. MR 31 \# 1326.

8a. Harish-Chandra, The characters of semisimple Lie groups, Trans. Amer. Math. Soc. 83 (1956), 98-163. MR 18, 318.

8b. __ Invariant eigendistributions on a semisimple Lie group, Trans. Amer. Math. Soc. 119 (1965), 457-508. MR 31 \#4862d.

9a. M. Hashizume, A. Kowata, K. Minemura and K. Okamoto, An integral representation of an eigenfunction of the Laplacian on the Euclidean space, Hiroshima Math. J. 2 (1972), 535-545.

9b. M. Hashizume, K. Minemura and K. Okamoto, Harmonic functions on hermitian hyperbolic spaces, Hiroshima Math. J. 3 (1973), 81-108. MR 50 \# 14073.

10a. S. Helgason, Differential operators on homogeneous spaces, Acta Math. 102 (1959), 239-299. MR 22 \#8457.

10b. Differential geometry and symmetric spaces, Academic Press, New York, 1962.

MR 26 \#2986.

10c. Radon-Fourier transforms on symmetric spaces and related group representations, Bull. Amer. Math. Soc. 71 (1965), 757-763. MR 31 \#3543.

10d. __ A duality for symmetric spaces with applications to group representations, Advances in Math. 5 (1970), 1-154. MR 41 \#858.

10e. _ The surjectivity of invariant differential operators on symmetric spaces. I, Ann. of Math. (2) 98 (1973), 451-479. MR 51 \#3804.

10f. __ Eigenspaces of the Laplacian; integral representations and irreducibility, J. Functional Analysis 17 (1974), 328-353. MR 51 \#3353.

10g. _ A duality for symmetric spaces with applications to group representations. II, Advances in Math. 22 (1976), 187-219.

10h. __ Some results on eigenfunctions on symmetric spaces and eigenspace representations, Math. Scand. (to appear).

11. A. Hole, Representations of nilpotent Lie groups on eigenspaces (preprint).

12. R. Hotta, On the realization of the discrete series for semisimple Lie groups, J. Math. Soc. Japan, 23 (1971), 384-407. MR 46 \#5531.

13. L.-K. Hua, Harmonic analysis of functions of several complex variables in classical domains, Science Press, Peking, 1958; English transl., Transl. Math. Monographs, vol. 6, Amer. Math. Soc., Providence, 1963. MR 23 \# A3277; 30 \#2162.

14a. M.-C. Hu, Determination of the conical distribution for rank one symmetric spaces, Thesis, Mass. Institute of Technology, 1973.

14b. Conical distributions for rank one symmetric spaces, Bull. Amer. Math. Soc. 81 (1975), 98-100. MR 51 \#6297. 
15. L. Hörmander, On the theory of general partial differential operators, Acta Math. 94 (1955), 161-248. MR 17, 853.

16. K. Johnson, Differential equations and an analog of the Paley-Wiener theorem for linear semisimple Lie groups, Nagoya Math. J. 64 (1976), 17-29.

17. K. D. Johnson and N. Wallach, Composition series and intertwining operators for the spherical principal series. I, Trans. Amer. Math. Soc. (to appear).

18. F. I. Karpelevic, The geometry of geodesics and the eigenfunctions of the Beltrami-Laplace operator on symmetric spaces, Trudy Moskov. Mat. Obšc. 14 (1965), 48-185 = Trans. Moscow Math. Soc., Amer. Math. Soc., Providence, R. I., 1967, pp. 51-199. MR 37 \#6876.

19. M. Kashiwara, A. Kowata, K. Minemura, K. Okamoto, T. Oshima and M. Tanaka, Eigenfunctions of invariant differential operators on a symmetric space (preprint).

20. A. A. Kirillov, Unitary representations of nilpotent Lie groups, Uspehi Mat. Nauk 17 (1962), 57-110 = Russian Math. Surveys 17 (1962), 53-104. MR 25 \# 5396.

21. A. Korányi and P. Malliavin, Poisson formula and compound diffusion associated to an overdetermined elliptic system on the Siegel half plane of rank two, Acta Math. 134 (1975), 185-209.

22a. B. Kostant, On the existence and irreducibility of certain series of representations, Bull. Amer. Math. Soc. 75 (1969), 627-642; and in Lie Groups and Their Representations (I. M. Gel'fand, Editor), Halsted Press (Wiley), New York, 1975, pp. 231-329. MR 39 \# 7031; 51 \# 5845 .

22b. Verma modules and the existence of quasi-invariant differential operators (Actes Colloq., Marseilles-Luminy, 1974), Lecture Notes in Math., vol. 466, Springer-Verlag, Berlin and New York, 1975, pp. 101-128.

23. J. Lepowsky, Conical vectors in induced modules, Trans. Amer. Math. Soc. 208 (1975), 219-272. MR 51 \# 12961.

24. J. B. Lewis, Eigenfunctions on symmetric spaces with distribution-valued boundary forms, $\mathbf{J}$. Functional Analysis (to appear).

25. D. B. Lowdenslager, Potential theory in bounded symmetric homogeneous complex domains, Ann. of Math. (2) 67 (1958), 467-484. MR 21 \#2836.

26. R. S. Martin, Minimal positive harmonic functions, Trans. Amer. Math. Soc. 49 (1941), 137-172. MR 2, 292.

27a. K. Minemura, Harmonic functions on real hyperbolic spaces, Hiroshima Math. J. 3 (1973), 121-151. MR 48 \# 11928.

27b. Eigenfunctions of the Laplacian on a hermitian hyperbolic space, Hiroshima Math. J. 4 (1974), 441-457.

27c. Eigenfunctions of the Laplacian on a real hyperbolic space, J. Math. Soc. Japan, 27 (1975), 82-105.

28. C. C. Moore, Compactifications of symmetric spaces. II. The Cartan domains, Amer. J. Math. 86 (1964), 358-378. MR 28 \#5147.

29. R. S. Palais, Seminar on the Atiyah-Singer index theorem, Princeton Univ. Press, Princeton, N. J., 1965. MR 33 \#6649.

30. R. Parthasarathy, Dirac operator and the discrete series, Ann. of Math. (2) 96 (1972), 1-30. MR 47 \#6945.

31. M. Rä̈, Solutions élémentaires des opérateurs différentiels bi-invariants sur un groupe de Lie nilpotent, C. R. Acad. Sci. Paris Sér. 273 (1971), A495-A498. MR 44 \#6908.

32. J. Rauch and D. Wigner, Global solvability of the Casimir operator, Ann. of Math. (2) 103 (1976), 229-236.

33. F. Rouvière, Sur la résolubilité locale des opérateurs bi-invariants, Ann. Scuola Norm. Sup. Pisa 3 (1976).

34. W. Schmid, Some properties of square-integrable representations of semisimple Lie groups, Ann. of Math. (2) 102 (1975), 535-564.

35a. T. O. Sherman, Fourier analysis on the sphere, Trans. Amer. Math. Soc. 209 (1975), 1-31.

35b. Fourier analysis on compact symmetric spaces; Local theory (preprint).

36. R. J. Stanton, On mean convergence of Fourier series on compact Lie groups, Trans. Amer. Math. Soc. 218 (1976), 61-87.

37. R. Takahashi, Sur les représentations unitaires des groupes de Lorentz généralisés, Bull. Soc. Math. France 91 (1963), 289-433. MR 31 \#3544. 
38. F. Trèves, Linear partial differential equations with constant coefficients: Existence, approximation and regularity of solutions, Gordon and Breach, New York, 1966. MR 37 \#557.

39. N. Wallach, On the Enright-Varadarajan modules, Ann. Sci. Ecole Norm. Sup. 9 (1976), 81-101.

40. B. Ørsted, Wave equations, particles and chronometric geometry, Thesis, Mass. Institute of Technology, 1976.

41. J. A. Wolf, Partially harmonic spinors and representations of reductive Lie groups, J. Functional Analysis 15 (1974), 117-154.

42. D. Wigner, Biinvariant operators on nilpotent Lie groups (preprint).

Department of Mathematics, Institute of Technology, Cambridge, Massachusetts 02139 